\title{
Nitric oxide synthase and background adaptation in Xenopus laevis
}

\author{
Wilfried Allaerts ${ }^{\mathrm{a}, *}$, Ruud Ubink ${ }^{\mathrm{a}}$, Jan de Vente ${ }^{\mathrm{b}}$, Rienk Tuinhof ${ }^{\mathrm{a}}$, Bruce G. Jenks ${ }^{\mathrm{a}}$, \\ Eric W. Roubos ${ }^{\text {a }}$ \\ ${ }^{a}$ Department of Cellular Animal Physiology, Nijmegen Institute for Neurosciences, University of Nijmegen, Toernooiveld 1, \\ 6525 ED Nijmegen, The Netherlands \\ ${ }^{\mathrm{b}}$ European Graduate School of Neuroscience (EURON), Maastricht University, Department of Psychiatry and Neuropsychology, P.O. Box 616, \\ 6200 MD Maastricht, The Netherlands
}

Received 22 November 1996; received in revised form 29 May 1997; accepted 11 September 1997

\begin{abstract}
Adaptation of the skin colour to the background light condition in the amphibian Xenopus laevis is achieved by migration of pigment granules in the skin melanophores, a process regulated by $\alpha$-MSH secretion from melanotrope cells in the pituitary pars intermedia (PI). $\alpha$-MSH secretion in turn, is regulated by various stimulatory and inhibitory messengers synthesized in brain nuclei, especially the hypothalamic suprachiasmatic and magnocellular nuclei and the locus coeruleus in the hindbrain.

In the present study, the roles in background adaptation of nitric oxide (NO) and NO synthase (NOS) enzyme activity were evaluated. In situ, using both immunohistochemistry with anti-human brain NOS (bNOS) serum in paraffin-embedded material and using nicotinamide adenine dinucleotide phosphate-diaphorase (NADPH-d) histochemistry in cryo-sections, we showed NOS in neurons in the optic tectum and in the locus coeruleus. NADPH-d reactivity was also found in neurons in the lateral amygdala, the ventral hypothalamic nucleus and in fibers in the median eminence. Using a Western blot stained with an anti-human bNOS serum, we demonstrated a $150 \mathrm{kDa}$ band in Xenopus hindbrain lysates, which is similar to the NOS protein present in the rat anterior pituitary, but which was not detectable in the lysates from both the neurointermediate and distal lobes in Xenopus. No differences in histochemical staining pattern or on Western blotting were observed between animals adapted to a black or a white background.

Paraffin sections of the endocrine PI and pars distalis did not reveal bNOS-like immunoreactivity. NADPH-d reactivity was observed in the endothelia of this gland. However, using a new procedure of thin cryo-sections of pituitary neurointermediate lobes, we observed bNOS-immunoreactive fibers as well as cyclic $3^{\prime}, 5^{\prime}$ guanosine monophosphate (cGMP)-accumulating fibers in the PI.

The PI may be regulated by NOergic neurons from higher brain centers. The possibility that NOergic neurons in the locus coeruleus are involved in the innervation of the PI needs further investigation. The latter neurons are probably not noradrenergic because double labeling studies show no co-localization of NADPH-d reactivity and tyrosine hydroxylase immunoreactivity in locus coeruleus neurons. (C) 1997 Elsevier Science B.V.
\end{abstract}

Keywords: bNOS-immunohistochemistry; NADPH-diaphorase staining; Cyclic GMP-accumulation; Pituitary pars intermedia; Locus coeruleus; Optic tectum * Corresponding author. Tel.: + 3124 3652705; fax: + 3124
3652714.

\section{Introduction}

Nitric oxide (NO), a free radical (Butler et al., 1995), has emerged as an intracellular and intercellular messenger molecule with a very wide range of biological roles. For instance, NO accounts for the biological 
activity of (or is) the endothelium-derived relaxing factor (EDRF) (Ignarro et al., 1987; Palmer et al., 1987; Garthwaite et al., 1988), acts as a retrograde messenger in memory formation (Schuman and Madison, 1991; Shibuki and Okada, 1991, Garthwaite and Boulton, 1995), is involved in cell-mediated immune responses in inflammation (Stuehr et al., 1989) and may regulate growth hormone $(\mathrm{GH})(\mathrm{Kato}, 1992)$ and luteinizing hormone $(\mathrm{LH})(\mathrm{Ceccatelli}$ et al., 1993) secretion from the pituitary. Furthermore, increased levels of NO synthase (NOS), protein and mRNA were detected in the rat anterior pituitary (AP) after gonadectomy (Ceccatelli et al., 1993) and in human pituitary adenomas (Lloyd et al., 1995), suggesting an important physiological role of NOS in the AP. In vivo NO is formed from L-arginine by each of the NOS isoenzymes, referred to as brain NOS (bNOS), endothelial NOS (eNOS) and macrophage NOS or inducible NOS (iNOS) (Garthwaite and Boulton, 1995). bNOS and eNOS are constitutively present and are activated through a calcium-dependent pathway (Garthwaite and Boulton, 1995), whereas iNOS is induced by immunostimulation and the activity of the latter enzyme is not dependent on increased intracellular calcium (Galea et al., 1992, Simmons and Murphy, 1992).

A main target for the messenger NO is to activate the cytoplasmic soluble form of guanylyl cyclase (sGC), stimulating the formation of cyclic $3^{\prime}, 5^{\prime}$ guanosine monophosphate (cGMP)(Bredt and Snyder, 1989; Knowles et al., 1989; de Vente et al., 1990; Garthwaite, 1991; Southam and Garthwaite, 1993).

Recently, the neuroanatomical distribution of NOSimmunoreactive neurons and the distribution of $\beta$ nicotinamide adenine dinucleotide phosphate-diaphorase (NADPH-d) positive neurons has been described in a number of amphibian (Brüning and Mayer, 1996; Muñoz et al., 1996; González et al., 1996) and reptilian species (Brüning et al., 1994; Smeets et al., 1997). Muñoz et al. (1996) suggest that the function of NO in the amphibian brain, considered to be primarily related to forebrain activities, may have been well preserved during vertebrate evolution (Allaerts et al., 1997).

Among amphibians, the South African clawed toad Xenopus laevis shows a particularly strong adaptive response to background light intensity. This adaptation involves activation of biosynthetic and secretory processes in the melanotrope cells of the pituitary PI, whereas these processes inactivate during white-background adaptation. Activation of the PI melanotropes results in the release of proopiomelanocortin (POMC)derived peptides, including $\alpha$-melanophore-stimulating hormone $(\alpha-\mathrm{MSH})$. In dermal melanophores, $\alpha-\mathrm{MSH}$ stimulates the dispersion of the black pigment melanin, thus causing skin darkening (Jenks et al., 1993; Roubos, 1997).
The aim of the present study was to investigate the possible role of $\mathrm{NO}$ in the control of background adaptation by Xenopus laevis. For this purpose, we studied the presence of the NOS enzyme in the pituitary and the brain using immunohistochemistry, NADPH-d histochemical staining and Western blotting. We also evaluated NOS activity during adaptation of Xenopus to a black or white background. Hereby, special attention was given to the brain centers involved in the regulation of the PI, viz. the hypothalamic suprachiasmatic and magnocellular nuclei and the locus coeruleus in the hindbrain (Tuinhof et al., 1994). Recent studies in amphibians, moreover, have corroborated the noradrenergic innervation of the PI from the locus coeruleus (Jansen et al., 1997). Co-localization studies of NADPH-d reactivity and tyrosine hydroxylase immunoreactivity (TH-IR) have been performed to compare the distribution of NOergic neurons with the aminergic innervation of the PI. Finally, we compared bNOS-immunoreactivity (bNOS-IR) and cGMP-IR in the pituitary PN and PI, following the procedure of de Vente et al. (1990; de Vente et al., 1996a,b).

\section{Materials and methods}

\subsection{Animals}

Adult Xenopus laevis weighing $\approx 25 \mathrm{~g}$ were obtained from laboratory stock. The toads were kept under constant illumination on either a black or a white background for at least 3 weeks $\left(22^{\circ} \mathrm{C}\right)$ and fed beef heart and trout pellets (Trouvit, Trouw, Putten, The Netherlands) once a week. Melanophore indices were estimated according to the protocol of Hogben and Slome (1931).

\section{2. bNOS-immunohistochemistry on paraffin-embedded tissue}

Toads were anaesthetized with $0.1 \%$ tricaine methane sulfonate (MS 222, Sandoz, Basle, Switzerland) and perfused via the heart with ice-cold $0.6 \%$ Ringer's solution for $3 \mathrm{~min}$, followed by perfusion for $15 \mathrm{~min}$ with either Bouin-Hollande or Zamboni fixative (Zamboni and De Martino, 1967). Brains and pituitaries of blackadapted and white-adapted toads were dissected and post-fixed for 3 or $16 \mathrm{~h}$ in either fixative. Brains and pituitaries were embedded in paraplast and sagittal sections of $10-20 \mu \mathrm{m}$ were cut with a Spencer 820 microtome (American Optical Instrument, Buffalo, NY). Sections were immunostained with the ABC method (Hsu et al., 1981), according to Ubink et al. (1994), with a polyclonal antiserum against human bNOS (Transduction, Lexington, KY; diluted 1:100) and the Vectastain ABC kit (Vector, Burlingame, CA; 
diluted 1:200). Dilutions of the antiserum were made in $0.01 \mathrm{M}$ sodium phosphate-buffered saline (PBS; pH 7.4) supplemented with $0.1 \%$ Triton X-100 (Sigma, St. Louis, MO) and $2 \%$ bovine serum albumin (BSA; Sigma), whereas the ABC reagent was diluted in PBS. Staining was accomplished by $30 \mathrm{~min}$ incubation with $0.04 \%$ diaminobenzidine (DAB; Sigma) and 0.015\% $\mathrm{H}_{2} \mathrm{O}_{2}$ in PBS, followed by a 5 min rinse in running tap water. Sections were mounted with Entellan (Merck, Darmstadt, Germany).

\subsection{NADPH-diaphorase histochemistry and \\ TH-immunohistochemistry on cryo-sections}

Black adapted and white adapted toads were anaesthetized and perfused as described above. Perfusion fixative was $2 \% \quad(w: v)$ freshly depolymerized paraformaldehyde in $0.05 \mathrm{M}$ sodium cacodylate buffer (pH 7.4), supplemented with $0.5 \%$ (v:v) glutaraldehyde and $0.5 \%(\mathrm{v}: \mathrm{v})$ picric acid after Tanaka et al. (1991). In another set of experiments, animals were perfused with periodate-lysine-paraformaldehyde (PLP) fixative after McLean and Nakane (1974), containing 2\% freshly depolymerized paraformaldehyde, $0.01 \quad \mathrm{M} \quad \mathrm{NaIO}_{4}$ (Merck) and $0.075 \mathrm{M}$ L-lysine (Sigma) in $0.0375 \mathrm{M}$ sodium phosphate buffer. The advantage of PLP fixation for cryo-immunohistochemistry lies in the crosslinking of carbohydrate moieties by lysine after oxidation by periodate (McLean and Nakane, 1974), yielding both good antigenicity and ultrastructural preservation (Allaerts et al., 1996). The brains and pituitaries were dissected and postfixed for $2 \mathrm{~h}$ at $4^{\circ} \mathrm{C}$ in the same fixative as used during perfusion, followed by cryoprotection in $30 \%(\mathrm{w}: \mathrm{v})$ sucrose in $0.15 \mathrm{M}$ phosphate buffer and frozen at $-70^{\circ} \mathrm{C}$. Sagittal and transversal sections of $15-20 \mu \mathrm{m}$ thick were cut on a HM 500-O cryostat (Microm; Adamas, Leersum, The Netherlands) and mounted on poly-L-lysine coated glass slides. For NADPH-d staining, $20 \mu \mathrm{m}$ thick cryosections were pre-incubated with $50 \mathrm{mM}$ Tris-buffered saline (TBS or Tris- $\mathrm{HCl}$; $\mathrm{pH}$ 7.6) for $30 \mathrm{~min}$ and incubated for $30 \mathrm{~min}$ at $37^{\circ} \mathrm{C}$ with $1 \mathrm{mM} \beta$-NADPH (Sigma) and $0.2 \mathrm{mM}$ nitroblue tetrazolium (Sigma) in $0.1 \mathrm{M}$ Tris $-\mathrm{HCl}$ buffer ( $\mathrm{pH}$ 7.6), supplemented with $0.2 \%$ Triton X-100 (Dawson et al., 1991). Sections were washed in 0.1 M PBS and mounted in 30\% (v:v) glycerol in 0.1 M PBS.

TH immunostaining was performed essentially as described before (Tuinhof et al., 1994), with minor modifications. Transversal cryo-sections of PLP-fixed Xenopus brains and pituitaries were immunostained using a monoclonal mouse anti-TH serum (Instar, Stillwater, MN) diluted 1:100 in PBS supplemented with $0.1 \%$ Triton X-100 and 2\% BSA (PBS-T). The secondary antiserum used was a FITC-labeled donkey anti-mouse IgG (Jackson Immunoresearch, West
Grove, PA) also diluted 1:100 in PBS-T. Co-localisation studies of NADPH-d and TH were performed on the same sections by combination of bright field and FITC-epifluorescence microscopy using a Leitz DM $\mathrm{RB} / \mathrm{E}$ microscope equipped with Leica Vario Orthomat E camera system.

\section{4. bNOS- and cGMP-immunohistochemistry on cryo-sections}

In another set of experiments, localization of bNOSIR, as well as cGMP-IR in cryosections of the brain and pituitary were investigated according to a procedure devised for studying cGMP-IR in brain slices (de Vente et al., 1990, 1996a,b). Briefly, adult Xenopus (7 white-adapted and 7 black-adapted animals) were decapitated, the brains and also some neurointermediate (NILs) and distal lobes were transferred into ice-cold incubation buffer ( $\mathrm{pH} 7.4$ ), aerated with $5 \% \mathrm{CO}_{2} / 95 \%$ $\mathrm{O}_{2}$. Subsequently, the brains were placed en bloc on a filter paper on a precooled stainless-steel table. Transverse $400 \mu \mathrm{m}$ thick slices are cut with a McIlwain tissue chopper, and separated from each other under a microscope, while being submerged in ice-cold incubation buffer aerated with $5 \% \mathrm{CO}_{2} / 95 \% \mathrm{O}_{2}$. Brain slices and pituitary lobes were transferred into incubation vials containing ice-cold, aerated buffer and then slowly equilibrated to $22^{\circ} \mathrm{C}$ under an atmosphere of $5 \% \mathrm{CO}_{2} /$ $95 \% \quad \mathrm{O}_{2}$. The phosphodiesterase (PDE) inhibitor isobutylmethylxanthine (IBMX; Sigma; $1 \mathrm{mM}$ ) was included from the beginning of the incubation. The NOdonor sodium nitroprusside (SNP; Sigma; $0.1 \mathrm{mM}$ ) was added after 10 min incubation. Thirty min after addition of the NO-donor, the incubations were terminated by adding ice-cold fixative solution, consisting of $4 \%$ paraformaldehyde (PFA) in $0.1 \mathrm{M}$ phosphate buffer (PB; pH 7.4). After $30 \mathrm{~min}$, slices and lobes were transferred into $4 \%$ PFA plus $10 \%$ sucrose in $0.1 \mathrm{M} \mathrm{PB}$ for another $90 \mathrm{~min}$, followed by a $30 \mathrm{~min}$ wash in ice-cold 0.1 M PB, containing $10 \%$ sucrose. Subsequently, they were embedded in Tissue-tek (Miles, Elkhart, IN) and quickly frozen using a Fast Inverted Cryo System (FICS, Adamas). A number of 6-10 $\mu \mathrm{m}$ thick sections were cut on a cryostat (Microm, Adamas) and thawed onto chrome-alum gelatin-coated slides.

Sections were processed for immunocytochemistry as described (de Vente et al., 1996a,b; Yamada et al., 1997). Antisera used were a polyclonal sheep anticGMP (Tanaka et al., 1997; diluted 1:4000), a polyclonal rabbit anti-cGMP (de Vente et al., 1996a,b; diluted 1:300) and a polyclonal sheep anti-bNOS (kindly provided by Dr P.C. Emson, U.K.; diluted 1:750). Dilutions of the sera were made in filtered TBS supplemented with $0.3 \%$ Triton X-100. The specificity of these antisera in staining mammalian tissues has 
been largely demonstrated (de Vente et al., 1996a,b; Herbison et al., 1996; Tanaka et al., 1997; Yamada et al., 1997). In the single cGMP-immunostaining procedure, secondary antisera used were the CY3-labeled goat anti-rabbit IgG (Jackson; diluted 1:800) or the FITC-labeled rabbit anti-sheep (Calbiochem, La Jolla, CA; diluted 1:150), depending on the host species of the primary antiserum. In the double immunolabeling procedure, rabbit anti-cGMP and sheep anti-bNOS were used as primary antisera and the secondary antisera were the CY3-labeled donkey anti-rabbit (Jackson; diluted 1:800) followed by the FITC-labeled rabbit antisheep IgG (Calbiochem; diluted 1:30). Co-localization was evaluated using combination of CY3- and FITCepifluorescence microscopy (Leitz DM RB/E).

\subsection{Western blotting}

Homogenates of NILs and distal lobes and of the hindbrain (HB) of Xenopus adapted to either a black or a white background were separated by sodium dodecyl sulfate (SDS)-gel electrophoresis, according to Laemmli (1970). Four NILs and distal lobes per experiment were homogenized with a teflon homogenizer in $20 \mu 1$ lysis buffer containing $0.1 \mu \mathrm{g} / \mathrm{ml}$ phenylmethylsulfonyl fluoride (PMSF; Sigma), $1 \mu \mathrm{g} / \mathrm{ml}$ trypsin inhibitor (type I-S; Sigma), 0.1\% (v:v) Triton X-100, 1\% (v:v) Tween20 and $0.1 \%$ (w:v) deoxycholic acid (Sigma) in $0.05 \mathrm{M}$ Hepes buffer. The HB, obtained by transversal cutting with a razor blade of the brain between telencephalon and myelencephalon, of one black- and one whiteadapted toad per experiment, was also homogenized in $200 \mu 1$ of the same lysis buffer. The homogenates were centrifuged at $12500 \times g$ for 5 min and diluted 1:1 with electrophoresis sample buffer containing 3\% (w:v) SDS, $10 \%$ (v:v) glycerol, $0.05 \%$ (w:v) bromophenol blue and $5 \%$ (v:v) 2-mercaptoethanol (Mizutani et al., 1994) in $60 \mathrm{mM}$ Tris buffer (pH 6.8). A 7.5\% acrylamide gel (in 0.375 M Tris; $\mathrm{pH}$ 8.8) was used as the resolving gel, with a stacking gel of $4.5 \%$ acrylamide (in $0.15 \mathrm{M}$ Tris; $\mathrm{pH}$ 6.8). After electrophoresis, the gels were used for western blotting at $10 \mathrm{~V}$ for $16 \mathrm{~h}$ to a nitrocellulose membrane (BA-S 85, Schleicher and Schuell, Dassel, Germany) in a wet transfer apparatus (Mini Trans-Blot Cell, Bio-Rad, Melville, NY), using transfer buffer containing $20 \%$ methanol, $0.192 \mathrm{M}$ glycine, $0.025 \mathrm{M}$ Tris and $0.1 \%$ SDS. The nitrocellulose membrane was stained using the polyclonal anti-human bNOS serum (Transduction; diluted 1:1000) and the enhanced chemiluminescence (ECL) reaction (ECL kit of Amersham Life Science, Buckinghamshire, UK)(Whitehead et al., 1979). As a positive control, a rat pituitary lysate obtained commercially (Transduction) was treated in the same way as the Xenopus samples and was also immunostained with the bNOS antiserum. Molecular mass markers were from a high molecular weight stan- dard mixture (range 30-200 kDa; Sigma) and were stained with $0.5 \%$ (w:v) amido black (Merck) in 1\% acetic acid.

\section{Results}

\subsection{Immunohistochemistry and enzyme histochemistry}

For paraffin immunohistochemistry, brains and pituitaries were fixed with Zamboni fluid or Bouin-Hollande. Using a polyclonal antiserum against human bNOS, we observed bNOS-like IR in neurons in the optic tectum (Fig. 1a) and bNOS-positive neurons and fibers in an area corresponding topographically to the locus coeruleus (Tuinhof et al., 1994) (Fig. 1b). However, in the paraffin-embedded pituitaries of these animals, no bNOS-like IR was found (Fig. 1c). No differences in bNOS-like immunostaining pattern of the optic tectum and locus coeruleus were observed between brains from Xenopus adapted to either black or white background for 3 weeks. In the brains of BouinHollande fixed Xenopus, the bNOS staining of neurons was less conspicuous than in the Zamboni-fixed Xenopus.

In order to verify the distribution of bNOS-like IR in the different brain and pituitary areas, NADPH-d histochemical staining was applied to $15-20 \mu \mathrm{m}$ thick cryosections of Xenopus brain and pituitary. Using NADPH-d staining, two grades of staining with the nitroblue tetrazolium reaction product were observed. Myelin-rich regions of the CNS showed no blue staining at all. A light blue staining was observed in myelinpoor areas of the brain and pituitary, whereas a dark blue staining pattern was present in neurons. The latter staining pattern was considered as NADPH-d positive staining. Darkly stained NADPH-d positive neurons occurred in the optic tectum (Fig. 2a), in the area corresponding to the locus coeruleus (Fig. 2b) and in the ventral hypothalamic nucleus (Fig. 2d). Beside the latter NADPH-d reactive areas, we recently described NADPH-d reactive neurons in the lateral and dorsal pallium and in the amygdala pars lateralis, in the nucleus anterodorsalis tegmenti and in the dorsal raphe nucleus (Allaerts et al., 1997). NADPH-d staining was negative in the magnocellular and suprachiasmatic nuclei of the hypothalamus. Except for some staining in endothelia, the pituitary neural, intermediate and distal lobes were NADPH-d negative in cryosections of animals fixed after Tanaka et al. (1991) (Fig. 2c). However, using cryosections of PLP-fixed animals (after McLean and Nakane, 1974), a slightly enhanced blue staining was noted in endothelia and some unidentified cells in the intermediate lobe (Fig. 2e). Strongly NADPH-d positive fibers were present in the median eminence (Fig. 2d). No cellular co-localization with TH 
immunoreactivity was found in neurons of the locus coeruleus, but some TH-immunoreactive neurons were found adjacent to NADPH- $\mathrm{d}^{+}$neurons in this area (compare Fig. 3a,b). These double stainings indicate that NOS and TH enzyme activities occur in different neurons, at least in the locus coeruleus.

We found no differences in NADPH-d reactivity between black-adapted or white-adapted animals, either in the pituitary or in the brain.
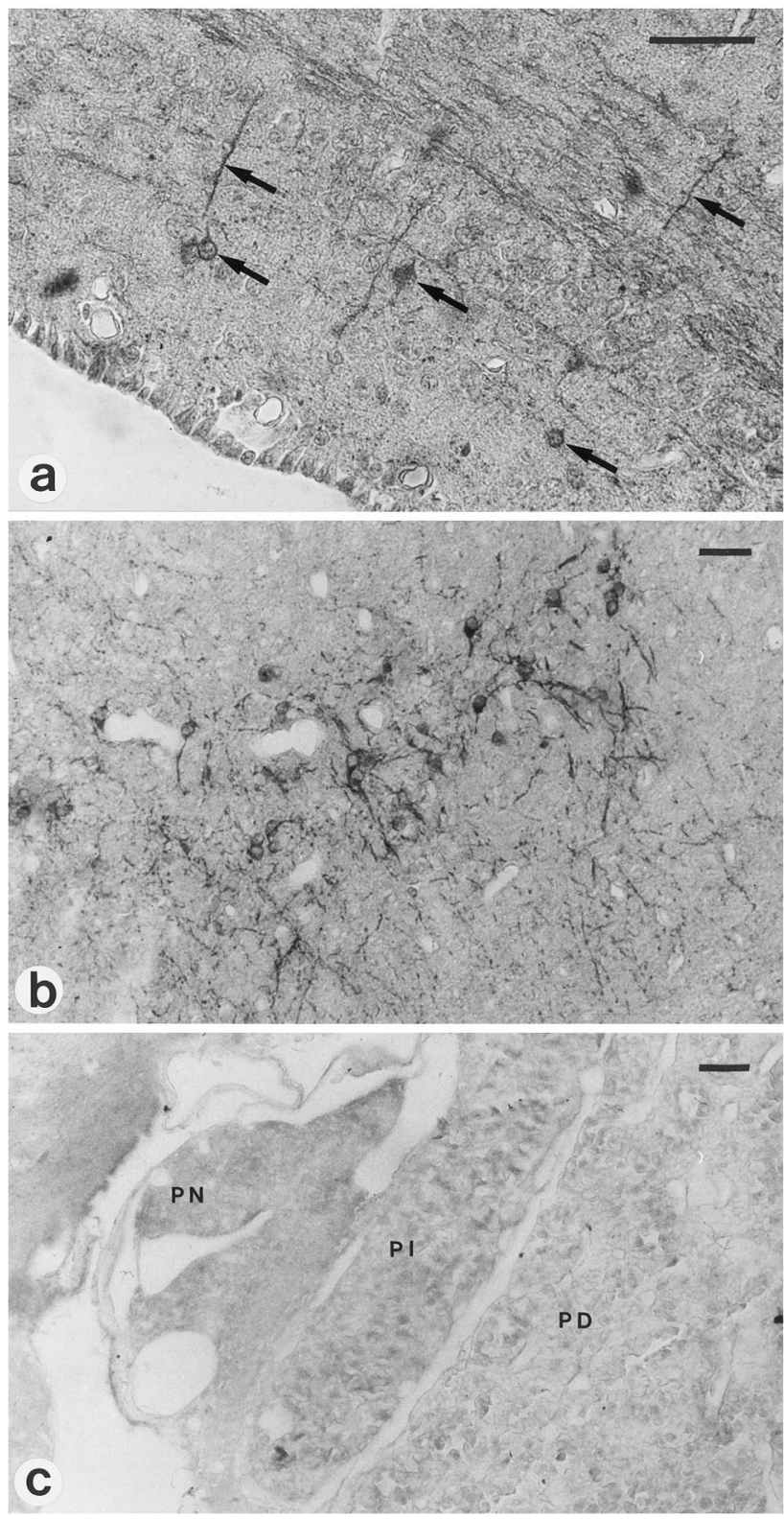

Fig. 1. Sagittal paraffin sections of black-adapted Xenopus laevis brain and pituitary, fixed with Bouin-Hollande (a) or Zamboni fluid $(b, c)$ and immunostained with polyclonal anti human bNOS serum, showing: (a) bNOS-like immunoreactive neurons in the optic tectum (arrows) $(\times 320)$; (b) bNOS-like immunoreactive neurons in an area corresponding topographically to the locus coeruleus $(\times 160)$; (c) absence of bNOS-like immunoreactivity in the pituitary $(\times 160)$. PI, pars intermedia; PN, pars nervosa; PD, pars distalis (or AP).(Scale bars $=50 \mu \mathrm{m})$.
In a final set of experiments, using $6-10 \mu \mathrm{m}$ thin sections of neurointermediate lobes, we observed clearly bNOS-positive fibers in the PI co-localized with cGMPIR fibers (Fig. 4a,b). When compared to the brain, the bNOS-IR neurons and fibers appeared to be distinct from but often in the immediate vicinity of the cGMPIR neurons and fibers (compare with Fig. 4c,d). The distribution of cGMP-IR neurons in the Xenopus brain is described elsewhere (Allaerts and de Vente, unpublished observations).

\subsection{Western blotting}

A Western blot stained with the polyclonal anti-human bNOS is shown in Fig. 5. Optimal separation was obtained using a $7.5 \%$ acrylamide gel and the optimal dilution of the bNOS antiserum for staining of the blot was 1:1000. Homogenates of NIL, distal lobe and hindbrain of Xenopus adapted to white and black backgrounds are electrophoretically separated in lanes 3-8. A commercial lysate from rat anterior pituitary (AP) was used as a positive control (lane 2). A mixture of six mass markers with MW ranging from 29 to $205 \mathrm{kDa}$ is shown in lane 1 . A high MW band corresponding to $\approx 150 \mathrm{kDa}$ was found in the rat AP lysate and in the Xenopus lysates of hindbrain (lanes 7 and 8). This high MW band was absent in the lysates of the Xenopus NILs and distal lobes. No effect of adaptation to a white (lanes $3,5,7$ ) or a black background (lanes 4, 6 , 8 ) was observed. An unidentified cross-reacting band at lower MW was stained in lane 2, i.e. the commercial rat AP lysate, which staining, however, was absent in the other lanes corresponding to Xenopus material.

\section{Discussion}

In the present study, we used immunohistochemical and enzyme histochemical staining procedures to detect NOS enzyme activities in the brain and pituitary of Xenopus laevis adapted to a black or a white background. We also compared the bNOS-IR in sections of conventional paraffin-embedded material with bNOSand cGMP-IR in thin cryosections of brain slices and pituitaries, processed according to a procedure devised for immunohistochemical visualization of cGMP-accumulation in brain slices (method of de Vente et al., 1990, 1996a,b). The latter procedure was found to be qualitatively superior to the paraffin method, with regard to both antigenicity and tissue preservation at the light-optical level, as for instance indicated by the lack of dehydration artefacts due to ethanol incubation. Using Western blotting of homogenates of NIL, distal lobe and hindbrain (including the metencephalon) of either black or white adapted Xenopus, we obtained 

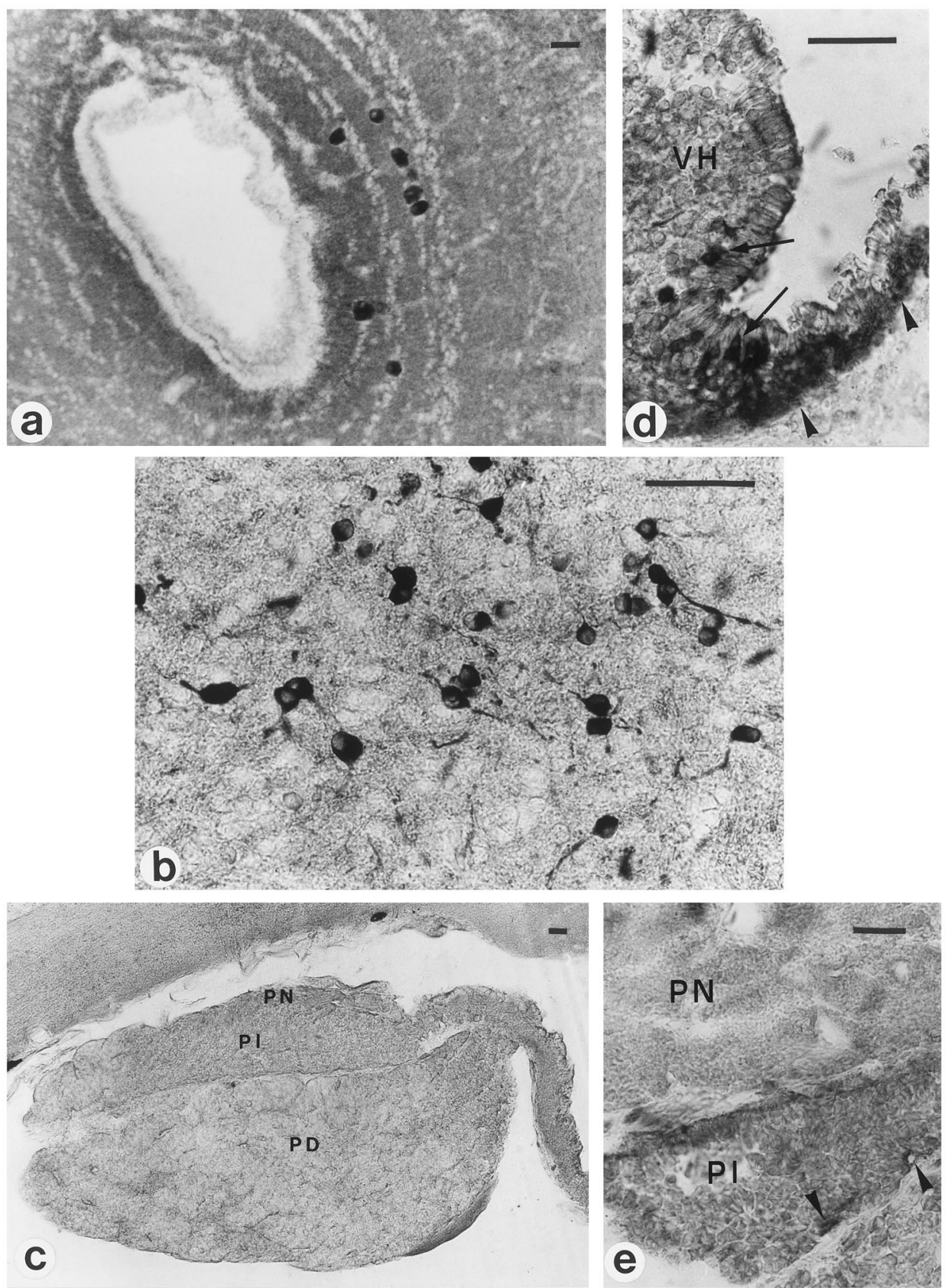

Fig. 2. Cryo-sections of Xenopus laevis brain and pituitary stained using the NADPH-diaphorase histochemical reaction: (a) white-adapted Xenopus fixed after Tanaka et al., 1991, showing NADPH-d ${ }^{+}$neurons in the optic tectum (sagittal; $\times 100$ ); (b) black-adapted Xenopus fixed with PLP after McLean and Nakane (1974), showing NADPH-d ${ }^{+}$neurons in the locus coeruleus (transversal; $\times 400$ ); (c) pituitary of black-adapted Xenopus fixed after Tanaka et al., 1991 (sagittal; $\times 63$ ); (d) detail of ventral hypothalamic nucleus $(\mathrm{VH})$ and median eminence in white-adapted Xenopus fixed with PLP, showing NADPH- ${ }^{+}$cells in the VH (long arrows) and NADPH- $\mathrm{d}^{+}$fibers in the median eminence (arrowhead)(sagittal; $\times 320$ );(e) detail of pituitary from white-adapted Xenopus fixed with PLP, showing faint NADPH-d ${ }^{+}$reaction in endothelia and some unidentified cells (arrowheads)(transversal; $\times 190)$. (Scale bars $=50 \mu \mathrm{m})$.

evidence for bNOS-like protein(s) in the brain of Xenopus, but were unable to demonstrate a bNOS-like protein in the NIL and distal lobe. This could be due to the limited number of NOergic fibers innervating the NIL. Moreover, our observation of bNOS-IR in fibers in the PI (although bNOS was not detected in the NIL lysates on Western blotting) corroborates the view that immunohistochemical detection in cryosections is the most sensitive of our methods used (Sternberger, 1979).

\subsection{Localisation of NOS-isozymes}

In paraffin sections we were able to demonstrate bNOS-like immunoreactive neurons in the optic tectum 
and locus coeruleus of Xenopus, using a polyclonal anti-human bNOS serum, whereas bNOS-IR was absent in the pituitary. In thin cryosections processed according to the method of de Vente et al., 1990, 1996a,b), apart from the regions reported bNOS-positive in paraffin sections and NADPH-d reactive in thick cryosections, bNOS-IR fibers were also detected in the pituitary PI and PN.

No differences were found in the expression of bNOS-like protein between black and white adapted animals. However, an adaptation period, of 3 weeks to a black or white background, may be inadequate to observe possibly transient changes in the bNOS expression in the brain following a change of background condition. A time series experiment will be undertaken to test this hypothesis.

Up to now, no evidence for a role of the optic tectum in the control of the melanotrope cells of Xenopus has been obtained. In contrast, the involvement of the locus coeruleus in the regulation of melanotrope cell activity is rather well documented (Tuinhof et al., 1994; see also below).

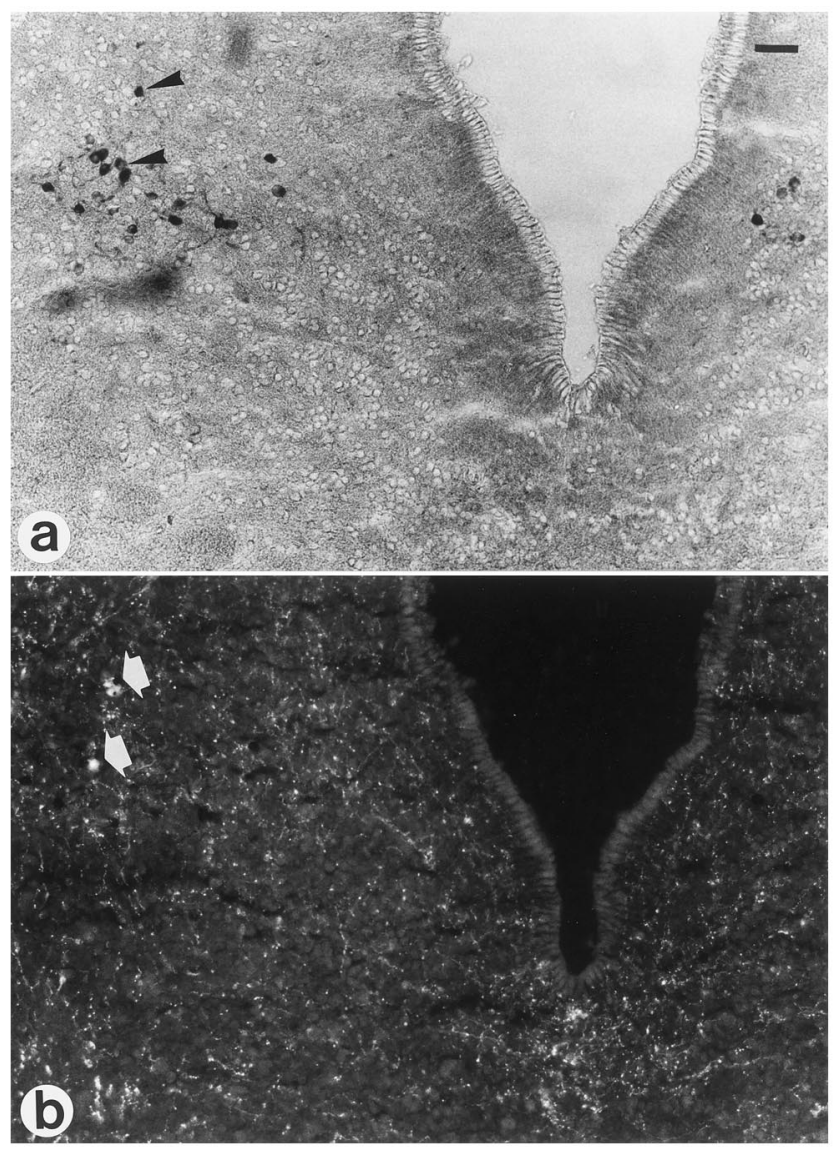

Fig. 3. Double labeling of transversal cryosection fixed with PLP of black adapted Xenopus laevis using NADPH-d staining (a) and anti-TH immunofluorescence-staining (b) in the locus coeruleus $(x$ 126). Note the NADPH-d-positive neurons (arrowheads in a) and TH-positive neurons (white arrowheads in b) in close juxtaposition. (Scale bar $=50 \mu \mathrm{m})$.
NADPH-d histochemical staining of cryo-sections showed darkly stained neurons in the optic tectum, the locus coeruleus, the ventral hypothalamic nucleus, the amygdala and fibers in the median eminence (present study and Allaerts et al., 1997). The distribution of NADPH-d ${ }^{+}$neurons corroborates the findings of Brüning and Mayer (1996) in Xenopus laevis and is also in line with recent studies in other amphibian species (González et al., 1996; Muñoz et al., 1996) and in some reptiles (Brüning et al., 1994; Smeets et al., 1997) (reviewed in Allaerts et al., 1997). In the rat, it was shown that NOS catalytic activity is responsible for NADPH-d staining (Hope et al., 1991; Dawson et al., 1991), although some tissues like the adrenal cortex and the liver display NADPH-d activity in the absence of NOS (Dawson et al., 1991). The faint NADPH-d reactivity in the PI of PLP-fixed Xenopus (except for the reactivity in some endothelial cells) may eventually be explained by assuming some cross-reactivity with mitochondrial cytochrome P450 reductase (Bredt et al., 1991), as was also suggested by Smeets et al. (1997) for some regions in the brain of Gekko gecko.

Our immuno- and enzyme-histochemical data demonstrate that NOS activity is present in some nuclei (locus coeruleus and possibly others), but not all nuclei involved in background adaptation in Xenopus laevis. Muñoz et al. (1996) observed NADPH-d activity in the suprachiasmatic and magnocellular nuclei in the frog Rana perezi, but this observation was not confirmed in studies in Xenopus laevis (Brüning and Mayer, 1996), nor in the urodele Pleurodeles waltl (González et al., 1996). Therefore, the data presented and also data from other studies indicate that the locus coeruleus neurons may be good candidates for the NOergic innervation of the PI.

\subsection{Relationship between $N A D P H-d^{+}$and $T H^{+}$ neurons in the locus coeruleus}

Retrograde labeling of the PI of Xenopus by Tuinhof et al. (1994), has shown that the area in the brainstem of Xenopus corresponding to the locus coeruleus in mammals, directly innervates the PI. Jansen et al. (1997) have corroborated these findings in the frog, Rana esculenta and the newt, Triturus carnifex and moreover, demonstrated colocalization of TH-IR and the retrograde label, indicating that noradrenergic neurons in the locus coeruleus are the source of noradrenergic projections to the PI. In order to test whether NOS is found in noradrenergic neurons, we performed co-localization studies of NADPH-d and TH. No colocalization of NADPH-d ${ }^{+}$with TH-IR neurons was observed in the locus coeruleus area, although both neuron types were closely intermingled, as described for Gekko gecko (Smeets et al., 1997). On the other hand, Muñoz et al. (1996) did find co-localization of 

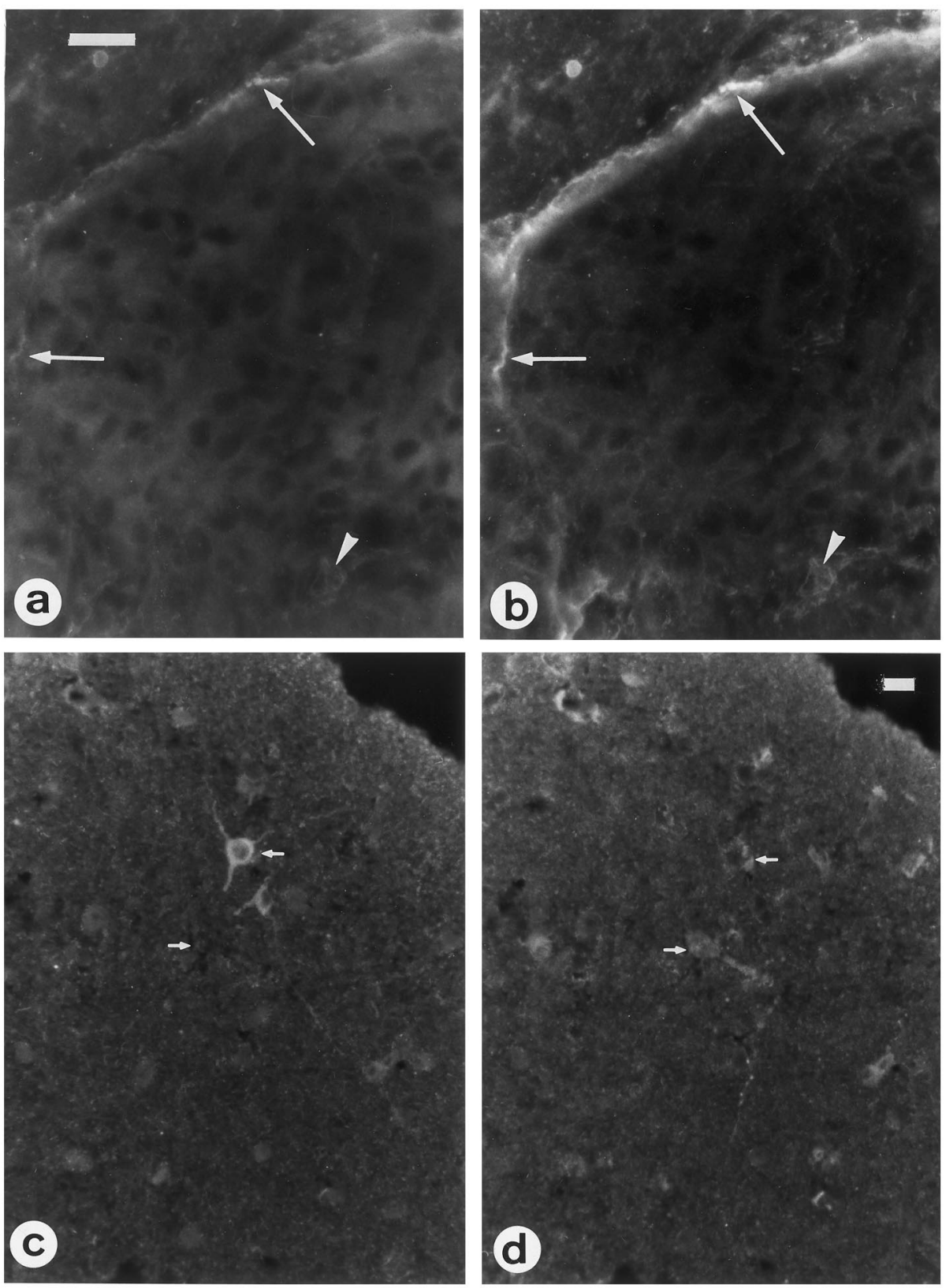

Fig. 4. Double labeling of $6 \mu \mathrm{m}$ cryosection of NIL (a, b) and brain (c, d) of a black-adapted Xenopus laevis processed according to the method of de Vente et al., 1990, 1996a,b. High magnification photographs of the PI $(a, b ; \times 500)$ and dorsal pallium $(c, d ; \times 250)$ revealing bNOS-IR (a, c; FITC optics) using a polyclonal sheep antiserum against bNOS (gift of Dr P.C. Emson; diluted 1:750) and revealing cGMP-IR (b, d; CY3 optics) using a polyclonal rabbit antiserum against cGMP (diluted 1:300). Note the co-localization of bNOS-IR and cGMP-IR in the PI in some fibers (long arrows) and in some unidentified PI cells (arrowheads), whereas in the dorsal pallium the bNOS-IR is topographically distinct from the cGMP-IR (small arrows indicate exactly matching positions). (Scale bar $=20 \mu \mathrm{m}$ ).

NADPH-d activity and TH-IR in the locus coeruleus of Rana perezi. According to Smeets et al. (1997), although extensive co-localization of NADPH-d or NOS and catecholamines occurs in the midbrain dopaminergic cell groups of reptiles and birds, co-localization is only 'sparsely' present in the corresponding cell groups of amphibians and mammals. Alternatively, it may be that the amounts of $\mathrm{TH}$ and NOS are inversely related within the neurons innervating the PI, making a demonstration of their co-localization technically difficult. A similar situation is observed with respect to the complementary expression of acetylcholinesterase and NADPH-d in the dorsal thalamic nuclei of the rabbit (Caballero-Bleda et al., 1991).

\subsection{Possible sources of NOS in the PI}

Apart from the bNOS-IR fibers in the PI and PN (Section 4), in cryosections of PLP-fixed pituitaries some NADPH-d activity was observed in endothelia of 
the pars distalis and PI, probably corresponding to eNOS. In the rat, Vanhatalo and Soinila (1995) found NADPH-d activity in nerve terminals and also in pituicytes in the PN and in cleft epithelial and interlobular cells (between the melanotropes) in the PI. Further research will be necessary to localize the NOergic neurons in higher brain centers that innervate the PI of Xenopus laevis and to investigate the NOergic input of the PI via fibers running through the median eminence. These fibers may appear similar to the NOergic nerve endings found in the mammalian PN (Kadowaki et al., 1994; Pow, 1994; Vanhatalo and Soinila, 1995), although due attention should be paid to the difference in pituitary innervation between mammals and amphibians. Also in analogy with the mammalian pituitary, a local (paracrine or autocrine) effect of NO in the PN (Pow, 1994) and the PI (Vanhatalo and Soinila, 1995) cannot be excluded, but requires further studies.

\section{4. cGMP-immunoreactivity in the brain and pituitary of Xenopus laevis}

In addition to the distribution of NOS-IR and NADPH-d positive neurons, we also observed cGMPIR in the brain and pituitary of Xenopus laevis. The distribution of cGMP-IR neurons and fibers in the brain corroborates the distribution of targets of the presumed NOergic signaling system (Allaerts and de Vente, unpublished observations). In particular, we found cGMP-IR fibers running through the ventral hypothalamus and median eminence towards the pituitary (not shown), as well as few cGMP-IR and

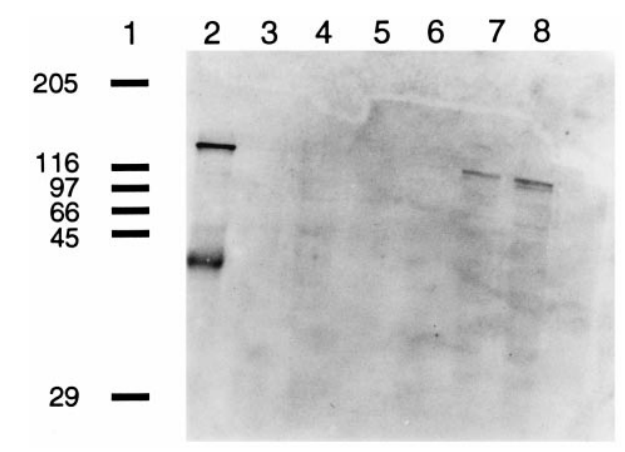

Fig. 5. Western blotting of SDS-PAGE gels with homogenates from Xenopus laevis NILs (lanes 3, 4), distal lobes (lanes 5, 6) and hindbrains (lanes 7,8). Animals are adapted to a white (lanes 3, 5 and 7) or a black background (lanes 4, 6 and 8). Gels are loaded with 10 $\mu 1$ lysate containing one equivalent of NIL or distal lobe per lane, or 1:40 equivalent of Xenopus laevis hindbrain per lane. A commercial lysate from rat pituitary ( $4 \mu \mathrm{g}$ protein $/ 10 \mu \mathrm{l})$, is used as a positive control in lane 2 . The blot is immunostained with the polyclonal anti human bNOS serum (diluted 1:1000). A high MW band corresponding to $\approx 150 \mathrm{kDa}$ is found in the rat anterior pituitary and Xenopus HB homogenates, but is absent from the Xenopus NIL and distal lobe homogenates.
bNOS-IR fibers in the PN and PI. In general, the distribution of bNOS-IR and cGMP-IR neuron somata and fibers showed distinct patterns, confirming the view of Southam and Garthwaite (1993) that in the rat, the microanatomical locations of cGMP-accumulation and NOS-activity are more complementary than identical: in some areas NOS occurs in postsynaptic structures and cGMP is accumulated in presynaptic elements (also in neuron fibers and glial cells), whereas in other areas the location was reversed (Southam and Garthwaite, 1993). The co-localization of bNOS-IR and cGMP-IR fibers in the pituitary PN and PI of Xenopus laevis, therefore contrasts with this principle of complementarity.

\section{Acknowledgements}

This work was enabled by a fellowship from the Human Capital and Mobility (HCM)-network of the European Community (Contract ERBCHRXCT920017) and grant number 805-26.241 from the Stichting Levenswetenschappen (SLW). We especially thank the suggestions of Dr Marco Buzzi (University of Ferrara, Italy) and Dr Hélène Castel (University of Rouen, France). Furthermore, we thank Dr Douglas W. Eib (University of Nijmegen) for the assistance with the Western blotting and Marianne Markerink-van Ittersum for the assistance with the cGMP-immunohistochemistry studies (University of Maastricht).

\section{References}

Allaerts, W., Fluitsma, D.M., Hoefsmit, E.C.M., Jeucken, P.H.M., Morreau, H., Bosman, F.T., Drexhage, H.A., 1996. Immunohistochemical, morphological and ultrastructural resemblance between dendritic cells and folliculo-stellate cells in normal human and rat anterior pituitaries. J. Neuroendocrinol. 8, 17-29.

Allaerts, W., Tuinhof, R., Ubink, R., Roubos, E.W., 1997. Nitric oxide synthase in the brain of the clawed toad Xenopus laevis: Is there a relationship with the visual system?. Belg. J. Zool. 127, $13-33$.

Bredt, D.S., Snyder, S.H., 1989. Nitric oxide mediates glutamatelinked enhancement of c-GMP levels in the cerebellum. Proc. Natl. Acad. Sci. 86, 9030-9033.

Bredt, D.S., Hwang, P.M., Glatt, C.E., Lowenstein, C., Reed, R.R., Snyder, S.H., 1991. Cloned and expressed nitric oxide synthase structurally resembles cytochrome P450 reductase. Nature 351, 714-718.

Brüning, G., Wiese, S., Mayer, B., 1994. Nitric oxide synthase in the brain of the turtle Pseudemys scripta elegans. J. Comp. Neurol. 348, 183-206.

Brüning, G., Mayer, B., 1996. Localization of nitric oxide synthase in the brain of the frog Xenopus laevis. Brain Res. 741, 331-343.

Butler, A.R., Flitney, F.W., Williams, D.L.H., 1995. NO, nitrosonium ions, nitroxide ions, nitrosothiols and iron-nitrosyls in biology: A chemist's perspective. Trends Pharmacol. Sci. 16, 18-22.

Caballero-Bleda, M., Fernandez, B., Puelles, L., 1991. Comparative mapping of acetylcholinesterase and reduced nicotinamide 
adenine dinucleotide diaphorase in the rabbit dorsal thalamus. Acta Anat. 140, 224-235.

Ceccatelli, S., Hulting, A.-L., Zhang, X., Gustafsson, L., Villar, M., Hökfelt, T., 1993. Nitric oxide synthase in the rat anterior pituitary gland and the role of nitric oxide in regulation of luteinizing hormone secretion. Proc. Natl. Acad. Sci. USA 90, 11292-11296.

Dawson, T.M., Bredt, D.S., Fotuhi, M., Hwang, P.M., Snyder, S.H., 1991. Nitric oxide synthase and neuronal NADPH diaphorase are identical in brain and peripheral tissues. Proc. Natl. Acad. Sci. USA 88, 7797-7801.

de Vente, J., Bol, J.G.J.M., Berkelmans, H.S., Schipper, J., Steinbusch, H.W.M., 1990. Immunocytochemistry of cGMP in the cerebellum of the immature, adult and aged rat: The involvement of nitric oxide: A micropharmacological study. Eur. J. Neurosci. $2,845-862$.

de Vente, J., Hopkins, D.A., Markerink-van Ittersum, M., Steinbusch, H.W.M., 1996a. Effects of the 3',5'-phosphodiesterase inhibitors isobutylmethylxanthine and zaprinast on NO-mediated cGMP accumulation in the hippocampus slice preparation: An immunocytochemical study. J. Chem. Neuroanat. 10, 241-248.

de Vente, J., Young, H.M., Steinbusch, H.W.M., 1996b. Immunohistochemical visualization of cyclic nucleotides. Methods Neurosci. $31,68-79$.

Galea, E., Feinstein, D.L., Reis, D.J., 1992. Induction of calcium-independent nitric oxide synthase activity in primary rat glial cultures. Proc. Natl. Acad. Sci. USA 89, 10945-10949.

Garthwaite, J., Charles, S.L., Chess-Williams, R., 1988. Endotheliumderived relaxing factor release on activation of NMDA receptors suggests roles as intercellular messenger in the brain. Nature 336, $385-388$.

Garthwaite, J., 1991. Glutamate, nitric oxide and cell-cell signalling in the nervous sytem. Trends Neurosci. 14, 60-67.

Garthwaite, J., Boulton, C.L., 1995. Nitric oxide signaling in the central nervous system. Annu. Rev. Physiol. 57, 683-706.

González, A., Muñoz, A., Muñoz, M., Marín, O., Arévalo, R., Porteros, A., Alonso, J.R., 1996. Nitric oxide synthase in the brain of a urodele amphibian (Pleurodeles waltl) and its relation to catecholaminergic neuronal structures. Brain Res. 727, 49-64.

Herbison, A.E., Simonian, S.X., Norris, P.J., Emson, P.C., 1996. Relationship of neuronal nitric oxide synthase immunoreactivity to GnRH neurons in the ovariectomized and intact female rat. J. Neuroendocrinol. 8, 73-82.

Hogben, L., Slome, D., 1931. The pigmentary effector system: The dual character of endocrine coordination in amphibian colour change. Proc. R. Soc. Lond. (Biol.) 108, 10-53.

Hope, B.T., Michael, G.J., Knigge, K.M., Vincent, S.R., 1991. Neuronal NADPH diaphorase is a nitric oxide synthase. Proc. Natl. Acad. Sci. USA 88, 2811-2814.

Hsu, S.M., Raind, L., Fanger, H., 1981. Use of avidin-biotin-peroxidase complex $(\mathrm{ABC})$ in immunoperoxidase technique. A comparison between $\mathrm{ABC}$ and unlabeled antibody (PAP) procedures. J. Histochem. Cytochem. 29, 577-580.

Ignarro, L.J., Buga, G.M., Wood, K.S., Byrns, R.E., Chaudhuri, G., 1987. Endothelium-derived relaxing factor produced and released from artery and vein is nitric oxide. Proc. Natl. Acad. Sci. USA 84, 9265-9269.

Jansen, K., Fabro, C., Artero, C., Feuilloley, M., Vaudry, H., Fasolo, A., Franzoni, M.F., 1997. Characterization of pars intermedia connections in amphibians by biocytin tract tracing and immunofluorescence aided by confocal microscopy. Cell Tissue Res. 287, 297-304.

Jenks, B.G., Leenders, H.J., Martens, G.J.M., Roubos, E.W., 1993. Adaptation physiology: The functioning of pituitary melanotrope cells during background adaptation of the amphibian Xenopus laevis. Zool. Sci. 10, 1-11.

Kadowaki, K., Kishimoto, J., Leng, G., Emson, P.C., 1994. Upregulation of nitric oxide synthase (NOS) gene expression together with NOS activity in the rat hypothalamo-hypophysial system after chronic salt loading: Evidence of a neuromodulatory role of nitric oxide in arginine vasopressin and oxytocin secretion. Endocrinology 134, 1011-1017.

Kato, M., 1992. Involvement of nitric oxide in growth hormone (GH)-releasing hormone-induced $\mathrm{GH}$ secretion in rat pituitary cells. Endocrinology 131, 2133-2138.

Knowles, R.G., Palacios, M., Palmer, R.M.J., Moncada, S., 1989. Formation of nitric oxide from L-arginine in the central nervous system: A transduction mechanism for stimulation of the soluble guanylate cyclase. Proc. Natl. Acad. Sci. USA 86, 5159-5162.

Laemmli, U.K., 1970. Cleavage of structural proteins during the assembly of the head of bacteriophage $\mathrm{T}_{4}$. Nature 227, 680-685.

Lloyd, R.V., Jin, L., Qian, X., Zhang, S., Scheithauer, B.W., 1995. Nitric oxide synthase in the human pituitary gland. Am. J. Pathol. 146, 86-94.

McLean, J.W., Nakane, P.K., 1974. Periodate-lysine-paraformaldehyde fixative: A new fixative for immunoelectron microscopy. J. Histochem. Cytochem. 22, 1077-1083.

Mizutani, F., Iwasawa, H., Tanaka, S., 1994. A morphometric analysis of the subcellular distribution of $\mathrm{LH} \beta$ and $\mathrm{FSH} \beta$ in secretory granules in the pituitary gonadotrophs of the frog (Rana japonica). Cell Tissue Res. 277, 417-426.

Muñoz, M., Muñoz, A., Marín, O., Alonso, J.R., Arévalo, R., Porteros, A., González, A., 1996. Topographical distribution of NADPH-diaphorase activity in the central nervous system of the frog Rana perezi. J. Comp. Neurol. 367, 54-69.

Palmer, R.M.J., Ferrige, A.G., Moncada, S., 1987. Nitric oxide release accounts for the biologic activity of endothelium-derived relaxing factor. Nature 327, 524-526.

Pow, D.V., 1994. Immunocytochemical evidence for a glial localisation of arginine, and a neuronal localisation of citrulline in the rat neurohypophysis: Implications for nitrergic transmission. Neurosci. Lett. 181, 141-144.

Roubos, E.W., 1997. Background adaptation by Xenopus laevis: A model for studying neuronal information processing in the pituitary pars intermedia. Comp. Biochem. Physiol. 119 A (in press).

Schuman, E.M., Madison, D.V., 1991. A requirement for the intracellular messenger nitric oxide in long term potentiation. Science 254, 1503-1506.

Shibuki, K., Okada, D., 1991. Endogenous nitric oxide release required for long-term synaptic depression in the cerebellum. Nature 349, 326-328.

Simmons, M.L., Murphy, S., 1992. Induction of nitric oxide synthase in glial cells. J. Neurochem. 59, 897-905.

Smeets, W.J.A.J., Alonso, J.R., González, A., 1997. Distribution of NADPH-diaphorase and nitric oxide synthase in relation to catecholaminergic neuronal structures in the brain of the lizard Gekko gecko. J. Comp. Neurol. 377, 121-141.

Southam, E., Garthwaite, J., 1993. The nitric oxide-cyclic GMP signalling pathway in the rat brain. Neuropharmacology 32, $1267-1277$.

Sternberger, L.A., 1979. Immunocytochemistry, 2nd edn. Wiley, New York.

Stuehr, D., Gross, S.S., Sakuma, I., Levi, R., Nathan, C.F., 1989. Activated murine macrophages secrete a metabolite of arginine with the bioactivity of endothelium-derived relaxing factor and the chemical reactivity of nitric oxide. J. Exp. Med. 169, 10111020.

Tanaka, J., Markerink-van Ittersum, M., Steinbusch, H.W.M., de Vente, J., 1997. Nitric oxide-mediated cGMP-synthesis in oligodendrocytes in the developing rat brain. Glia 19, 286-297.

Tanaka, S., Nomizu, M., Kurosomi, K., 1991. Intracellular sites of proteolytic processing of pro-opiomelanocortin in melanotrophs and corticotrophs in rat pituitary. J. Histochem. Cytochem. 39, $809-821$. 
Tuinhof, R., Artero, C., Fasolo, A., Franzoni, M.F., Ten Donkelaar, H.J., Wismans, P.G.P., Roubos, E.W., 1994. Involvement of retinohypothalamic input, suprachiasmatic nucleus, magnocellular nucleus and locus coeruleus in control of melanotrope cells of Xenopus laevis: A retrograde and anterograde tracing study. Neuroscience 61, 411-420.

Ubink, R., Halasz, N., Zhang, X., Dagerlind, Å., Hökfelt, T., 1994. Neuropeptide tyrosine is expressed in ensheating cells around the olfactory nerves in the rat olfactory bulb. Neuroscience 60,709-726.

Vanhatalo, S., Soinila, S., 1995. Hypothalamic nitric oxide-containing pituitary projecting pathways. J. Chem. Neuroanat. 8, 165-173.
Whitehead, T.P., Kricka, L.J., Carter, T.J.N., Thorpe, G.H.G., 1979. Analytical luminescence: Its potential in the clinical laboratory. Clin. Chem. 25, 1531-1546.

Yamada, K., Xu, Z.-Q., Zhang, X., Gustafsson, L., Hulting, A.-L., de Vente, J., Steinbusch, H.W.M., Hökfelt, T., 1997. Nitric oxide synthase and cGMP in the anterior pituitary gland: Effect of a GnRH antagonist and nitric oxide donors. Neuroendocrinology 65 , $147-156$.

Zamboni, L., De Martino, C., 1967. Buffered picric acid-formaldehyde: A new, rapid fixative for electron microscopy. J. Cell Biol. 35, 148A. 